\title{
Sutureless mitral valve replacement with bioprostheses and Nitinol attachment rings: Feasibility in acute pig experiments
}

\author{
Eric Berreklouw, MD, PhD, ${ }^{a}$ Sergey Leontyev, MD, ${ }^{\mathrm{b}}$ Susann Ossmann, vet med, ${ }^{\mathrm{b}}$ Christian Velten, Ing, ${ }^{\mathrm{c}}$ \\ Bernd Vogel, Ing, ${ }^{\mathrm{c}}$ Stephan Dhein, MD, PhD, ${ }^{\mathrm{b}}$ and Friedrich W. Mohr, MD, $\mathrm{PhD}^{\mathrm{b}}$
}

Objective: There is a need for fast, minimally invasive sutureless replacement of mitral valves.

\begin{abstract}
Methods: Unchanged FDA-approved biological valve prostheses were sutured to Nitinol attachment rings (Endosmart, Stutensee, Germany) that were covered with textile (devices). The lower flange of the device was stretched in ice water and maintained in a stretched position with stretching sutures. In 9 acute pig experiments through a limited left thoracotomy, a single suture was placed around the mitral annulus, the device was positioned, the annular suture tied, the stretching sutures retracted, and the device activated by warm saline. Position of the device, heart and valve function, coronary arteries, left ventricular outflow tract, and surrounding structures were observed with transoesophageal echocardiography, left ventricular and coronary angiograms, and pathologic examination at autopsy.
\end{abstract}

Results: The devices could be easily navigated to the mitral valve annulus and actuated within seconds. Three devices were placed with warm blood in the operative field and were tilted or dislocated at autopsy. In the other 6 devices, transesophageal echocardiography and left ventricular and coronary angiography demonstrated normal prosthetic valve and heart function, without valvular or para-device leakage, and with normal aortic valve and coronary arteries. At autopsy in these 6 cases, the devices were strongly fixed at the anatomic mitral valve annulus, without abnormalities of the device, heart valve prosthesis, left ventricular outflow tract, or aortic valve ostium.

Conclusions: Nitinol attachments rings combined with unchanged biological valve prostheses can make fast and strong sutureless replacement of the mitral valve feasible in acute pig experiments. Applicators that constrain and release the device mechanically need to be developed. (J Thorac Cardiovasc Surg 2011;142:390-5)

Video clip is available online.

Hand suturing is the current standard of attaching a mitral valve prosthesis to the anatomic mitral valve annulus. However, this method consumes relatively a great deal of time, particularly in multivalve and combined procedures, and makes minimally invasive valve surgery less favorable. Currently, there is renewed interest in sutureless valve implantations, mostly by mounting a biological aortic valve into a metal stent and compressing it into a catheter sleeve. ${ }^{1,2}$ However, with this technique the diseased valve

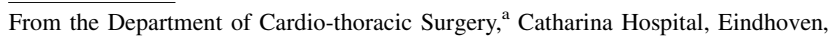
The Netherlands; the Animal Laboratory, ${ }^{\mathrm{b}}$ Herzzentrum Leipzig, Leipzig, Germany; and Endosmart GmbH, ${ }^{\mathrm{c}}$ Stutensee, Germany.

Disclosures: Authors have nothing to disclose with regard to commercial support.

Received for publication Sept 16, 2010; revisions received Oct 24, 2010; accepted for publication Dec 16, 2010; available ahead of print Feb 7, 2011.

Address for reprints: Eric Berreklouw, MD, PhD, Department of Cardio-thoracic Surgery, Catharina Hospital. PO Box 1350, 5602 ZA, Eindhoven, The Netherlands

(E-mail: eric.berreklouw@catharina-ziekenhuis.nl).

0022-5223/\$36.00

Copyright (C) 2011 by The American Association for Thoracic Surgery

doi: $10.1016 /$ j.jtcvs.2010.12.018

is not removed, and current valved stents do not result in outcomes comparable with surgically removed and replaced heart valves. ${ }^{3}$ So far, there have been no reports about the clinical use of primary mitral valved stents, but there have been a few reports about their experimental use. $^{4-6}$ Previously, we ${ }^{7}$ have shown that it is feasible to use Nitinol attachment rings to attach mechanical aortic valve prostheses solidly to the aortic valve annulus after removal of the original aortic valve in acute pig studies; we observed that such rings can withstand a high pulling force. Recently, $\mathrm{we}^{8}$ found that these valved rings remained well fixed and without leakage in the aortic position in pigs during more than 90 days of follow-up. With this study we wanted to test the feasibility of similar sutureless Nitinol rings to attach unchanged Food and Drug Administration (FDA)-approved biological valve prostheses at the anatomic mitral valve annulus. Because proven long-term durability is an important feature of most available FDAapproved biological valve prostheses, our intention was not to design a complete new heart valve prosthesis but just to provide a new sutureless implantation method.

\section{MATERIALS AND METHODS}

\section{Devices and Applicator}

After extensive in vitro testing, a mitral valve attachment ring (MVAR) was manufactured from Nitinol memory metal (Endosmart GmbH, Stutensee, Germany) and covered with vascular graft textile (knitted fabric $100 \%$ polyester; Jotec $\mathrm{GmbH}$, Hechingen, Germany). This proprietary ring was 

Abbreviations and Acronyms
ASD $=$ atrial septal defect
FDA $=$ Food and Drug Administration
$\mathrm{LV}=$ left ventricular
LVOT $=$ left ventricular outflow tract
MVAR $=$ mitral valve attachment ring
TEE $=$ transesophageal echocardiography

given a slightly sinusoidal shape to match the upside of a Perimount biological valve prosthesis (Edwards Lifesciences, Inc, Irvine, Calif) and possessed a fixed upper and flexible lower flange. The unchanged FDAapproved valve prosthesis ( 7 times a reversed $23-\mathrm{mm}$ aortic prosthesis and 2 times a $25-\mathrm{mm}$ mitral valve prosthesis) was placed on top and through the MVAR, and the textile of both devices was fixed together with a running nonresorbable polypropylene suture (4-0 Prolene; Ethicon, Inc, Somerville, NJ) (Figure 1, $A$ and $B$ ). An applicator was manufactured from stainless steel, with a flexible Nitinol handle (Pierre Janssen, Instrumentation Department Catharina Hospital, Eindhoven, The Netherlands). The MVAR with valve prosthesis (device) was loaded on the applicator with 3 temporary fixing sutures. The lower flange of the MVAR was composed of separate fingers that were temporary stretched manually in iced saline to facilitate passage of the device through the mitral valve annulus. To prevent unintended early expansion of the stretched lower flange during navigation, we applied 3 temporary stretching sutures through the textile of the lower flange, led them through the valve, and fixed them to the applicator. The applicator loaded with the device remained in iced saline until its use (Figure E1). Once the device was in place at its desired position at the mitral valve annulus, it was activated by being flushed with warm (about $45^{\circ} \mathrm{C}$ ) saline from a syringe. During activation the annulus was clamped between the expanding lower flange and fixed upper flange (Video 1). Similar rings were combined with unchanged FDA-approved mechanical mitral valve prostheses (St Jude Medical Inc, St Paul, Minn) and successfully tested in in vitro pig hearts in the mitral position (Video 2), but is was decided to proceed first with the combined biological valved device, because in future long-term testing no or limited anticoagulation was preferred to enhance the likelihood of late animal survival.

\section{Surgical Procedures and Investigations}

At the start of the surgical procedure, the valve prosthesis was hand sewn to the MVAR. The device was loaded on the applicator, manually stretched in iced saline, and the lower flange temporarily kept in a stretched position by stretching sutures. In 9 anesthetized and ventilated female German Saddleback pigs (weighing between 61 and $74 \mathrm{~kg}$ ), a limited left-sided thoracotomy was performed through the fourth or fifth intercostal space. One rib was partially removed, and more ribs were mobilized if needed. The pericardium was opened longitudinally and retracted. After heparinization, the animal was placed on full bypass with arterial cannulation in the descending aorta $(6 \times)$, right femoral artery $(2 \times)$, or left carotid artery $(1 \times)$. Venous cannulation was done through the right atrial appendage. A needle was placed into the ascending aorta, the aorta was crossclamped, and cold crystalloid $(1 \times)$ or blood cardioplegic $(6 \times)$ solution was administered. In the first and third case, there was a deviation from the protocol by fibrillating the heart with rapid pacing. The lateral wall of the left atrium was opened longitudinally and retracted by a retractor. The mitral valve in a pig, as approached from the left side, showed to be more in a more vertical plane and more clockwise rotated than in humans, as approached from the right side, with for the surgeon the posterior leaflet on the left and the anterior leaflet on the right. In the first 3 cases the mitral valve leaflets were left intact. In the last 6 cases the anterior mitral leaflet and its attaching chordae were resected. A 2-0 running nonresorbable polyester 2-0 suture (Topline PH26; HP Medica GmbH, Augsburg, Germany) was placed around mitral annulus, with a small felt patch at the start and end of the suture. This was done not only because the annular tissue in these young and healthy pigs was very flexible, but also because only one size MVAR prototype was available. In 2 cases the device needed to be repositioned. In the first 5 cases no guiding wires were used or left behind. In the last 4 cases, 1 or 2 guiding sutures were used between the mitral valve annulus and the upper flange of the device, which were tied after activation of the device in only 1 case. In all other cases in which guiding wires were used, those were removed after activation. The applicator loaded with the device was navigated to the mitral valve annulus, and after inspection of its correct position, the annular suture was pulled and tied, and the 3 temporary stretching sutures were cut and retracted (Figure E2, $A$ ). Warm $\left(45^{\circ} \mathrm{C}\right.$ ) saline was flushed over the device with a $30-\mathrm{mL}$ syringe. This was done twice, although one time seemed to be appropriate too (Figure E2, B). After the position of the device and the good function of the valve blades had been checked, in the last 4 cases a left ventricular (LV) vent was left behind through the valve for LV decompression and de-airing. The left atrium was closed with 2 layers of running 4-0 Prolene polypropylene sutures over felt strips (C. R. Bard, Inc, Covington, Ga), and the aortic crossclamp was removed. The heart was defibrillated as necessary (Video 3 ). After adequate reperfusion the left vent was removed. A summary of the surgical procedures is given in Table 1. Inotropics and antiarrhythmic drugs were given as needed, the animal was weaned from the extracorporeal circulation, and the calculated dose of protamine was given. Heart and valve function were evaluated and registered by transesophageal echocardiography with Doppler (TEE) before bypass, before weaning off bypass, and after

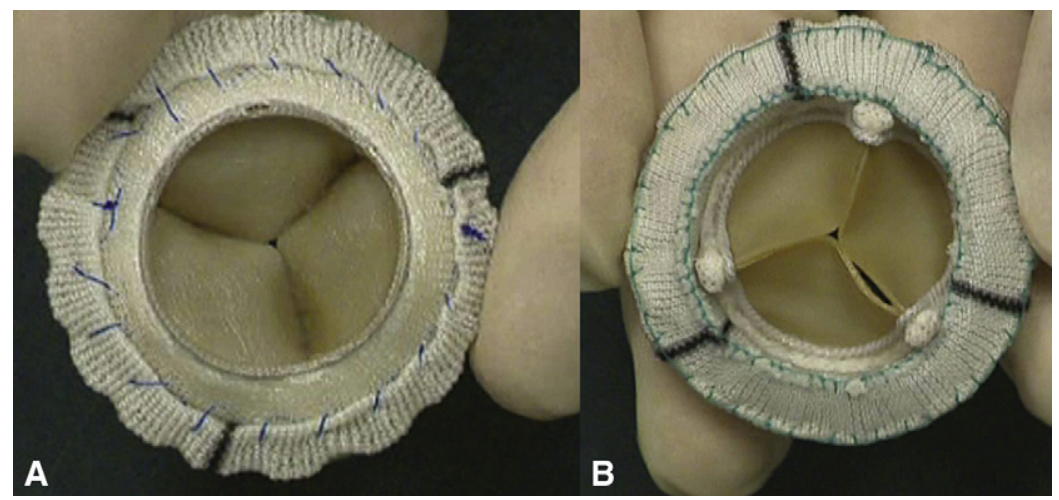

FIGURE 1. A, Mitral valve attachment ring (MVAR) with biological valve prosthesis (device) from above, left atrial side. B, MVAR with biological valve prosthesis (device) from below, left ventricular side. 
TABLE 1. Procedures

\begin{tabular}{|c|c|c|c|c|c|c|c|}
\hline Case no. & Arterial canulation & $\begin{array}{c}\text { Cardiac } \\
\text { protection }\end{array}$ & $\begin{array}{c}\text { AML } \\
\text { removed }\end{array}$ & $\begin{array}{c}\text { Annular } \\
\text { suture }\end{array}$ & $\begin{array}{c}\text { Guiding } \\
\text { suture }\end{array}$ & $\begin{array}{c}\text { Guiding } \\
\text { suture tied }\end{array}$ & Heart harvesting \\
\hline 1 & Desc aorta & $\mathrm{VF}^{*}$ & No & Yes & No & No & Thoracotomy \\
\hline 2 & Desc aorta & $\mathrm{CC}$ & No & Yes & No & No & Thoracotomy \\
\hline 3 & Femoral art & $\mathrm{VF}^{*}$ & No & Yes & No & No & Thoracotomy \\
\hline 4 & Femoral art & $\mathrm{BC}$ & Yes & Yes & No & No & Sternotomy \\
\hline 5 & Carotid art & $\mathrm{BC}$ & Yes & Yes & No & No & Sternotomy \\
\hline 6 & Desc aorta & $\mathrm{BC}$ & Yes & Yes & 1 & Yes & Sternotomy \\
\hline 7 & Desc aorta & $\mathrm{BC}$ & Yes & Yes & 2 & No & Sternotomy \\
\hline 8 & Desc aorta & $\mathrm{BC}$ & Yes & Yes & 2 & No & Sternotomy \\
\hline 9 & Desc aorta & $\mathrm{BC}$ & Yes & Yes & 2 & No & Sternotomy \\
\hline
\end{tabular}

$V F$, Ventricular fibrillation; $C C$, cold crystalloid cardioplegia; $B C$, cold blood cardioplegia; desc, descending; art, artery; $A M L$, anterior mitral valve leaflet; $L V$, left ventricle. *Deviation from protocol; operated on fibrillating heart.

bypass. After bypass, the animal was kept alive for another 2 to 3 hours. In this time, and/or during reperfusion, an LV angiogram was done through the right femoral artery, and any mitral valve regurgitation was graded to be absent or, if existent, from $1+$ to $4+{ }^{9}$ A nonselective supravalvular coronary angiogram was done to exclude any damage to the coronary arteries or aortic valve. All angiograms were evaluated by an independent cardiologist (Yaroslava Dmitrieva, MD), who has not been involved in the operative procedures. After this time, each animal was humanely killed, and the heart was taken out for gross pathologic examination. In the first 3 cases, the heart was harvested through the limited left-sided thoracotomy and, in the last 6 cases, through a separate sternotomy. At gross pathologic examination, position and function of the device were determined. Paravalvular leakage was sought for with 1-mm probes. Inspection for damage of surrounding structures included inspection of the LV outflow tract (LVOT) and aortic valve ostium. Pictures were taken from all specimens. All experiments were approved and performed along the Animal Research Ethics regulations of the institution and country where the experiments took place. In all experiments the animals received care in compliance with the European Convention on Animal Care and the Guide for the Care and Use of Laboratory Animals as published by the US National Institutes of Health (NIH Publication No. 85-23, revised 1996).

\section{RESULTS}

In all cases the device could be navigated quite easily to the mitral valve annulus, and activation by heating did occur within a few seconds. Once in the right position at the annulus, the device attached itself solidly. There were 2 instances in which there was a deviation from the operative protocol. In the first case the procedure was intentionally performed on a fibrillating heart. In that case the device showed a tendency to expand too early, owing to contact with warm blood. It was necessary to reposition the device, and finally its suboptimal and tilted position was wrongly accepted. In the third case, severe bleeding at the aortic needle hole did occur, and the operation was continued with a fibrillating heart. In the eighth case, a large atrial septal defect (ASD) was encountered, which was primarily closed with a single layer of large-bite suture, resulting in difficulties to attach the device at its lowest point at the mid-posterior side of the distorted annulus. In the other 6 cases, in which cold cardioplegic arrest was used and a dry operative field was obtained, there were no difficulties to navigate, position, and activate the device at the mitral annulus. Although it was difficult to retract some of the temporary stretching sutures, in all cases the activation with warm saline was instant, and this did not hinder the full expansion of the device and removal of the applicator. In all cases done under cardioplegic arrest and with a dry operative field, the position of the device was verified by the surgeon to be at the right place at the mitral valve annulus. In 8 cases the animals could be weaned off the extracorporeal circulation (ECC). In the 6 cases in which the aorta was crossclamped, and excluding the ASD case, the average crossclamp time was $29.8 \mathrm{~min}-$ utes. The navigation of the device and its activation took less than about 1 minute. The rest of the crossclamp time was consumed by taking out the anterior mitral leaflet, placing the annular suture, checking the correct position of the device, placing the LV vent, and closing the atrial wall with a double-layered suture over Teflon strips. In the second case, there was a long period of low cardiac output, most likely owing to air embolism. In the third case, the arterial cannula in the groin was accidentally removed early during the operation and needed to be reinserted in the ascending aorta, during which the animal sustained a period of shock. The animal could be weaned off the ECC but died within a half hour thereafter. In 8 cases a TEE was obtained that showed normal function of the valve prosthesis in 6 , without any important mitral valve regurgitation, para-device leakage, or LVOT obstruction (Figure 2, $A$ and $B$ ). The average mean gradient over the valve was $3.4 \mathrm{~mm} \mathrm{Hg}$, with an average maximum gradient of $6.9 \mathrm{~mm} \mathrm{Hg}$. In the first case that was done under ventricular fibrillation a $2+$ regurgitation was seen, whereas in the eighth case with an ASD the device was seen in the left atrium. LV (Figure 3, $A$ and $B$ ) and coronary angiography, as done in 5 cases, demonstrated the position of the device at the mitral valve annulus in all, without any mitral valve regurgitation, para-device leakage, and with normal LVOT and coronary anatomy. At autopsy, in all the cases in which cold cardioplegic arrest was used, with the exception of the ASD case, the device proved to be solidly fixed to the anatomic mitral valve annulus, 


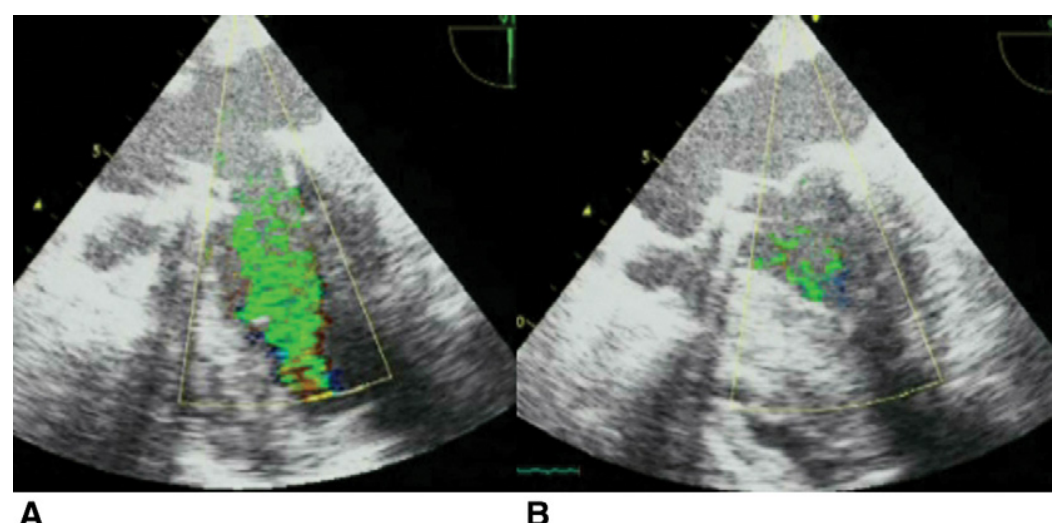

FIGURE 2. Device in mitral position at diastole (A) and systole (B) on transesophageal echocardiography.

without any para-device leakage, with normal prosthetic valve function, and no damage to the surrounding tissues, including a widely patent LVOT and aortic valve ostium (Figure 4, $A$ and $B$; Video 4). In the first case the device was found tilted at the posterior side, as was seen during implantation. In the third case the device was found to be migrated in the left atrium. In both cases there had been a deviation from the protocol, working in a warm fibrillating heart, that led to a too early expansion of the lower flange. In the eighth case the device was found to be migrated in the left atrium and the ASD was found to have reopened again. The outcome of the experiments is summarized in Table 2 .

\section{COMMENT}

Although our experience with the use of Nitinol attachment rings to attach unchanged FDA-approved biological valve prostheses suturelessly to the mitral valve annulus is still very limited, we demonstrated that it is potentially feasible to replace the mitral valve with such a device in an arrested heart, without any para-device or valvular leakage, low valve gradients, and no damage to the surrounding structures. At autopsy, in the first case a tilted position and in the third and eighth cases a luxated position of the device were found. In 2 of these cases there had been a devia- tion from the study protocol by trying to implant the device in a warm fibrillating heart. Not only should we have stuck to the protocol, but the imperfect position should not have been accepted by the surgeon. That the TEE and LV angiogram before the animals were humanely killed did not show any regurgitation in the first and third cases can be explained by the original mitral valve being intact. In the eighth case the anatomy of the mitral annulus had been distorted after closure of a large ASD. Also in this case the operative field was hindered by warm blood. In all 3 cases the stretching sutures could not prevent early expansion of the lower flange after contact with the warm heart and blood. A stronger mechanical way of constraining the lower flange may prevent such early expansion of the lower flange and could make use of the device in a warm beating or fibrillating heart possible. One can argue that placing a valve prosthesis on top and through an intra-annular attachment ring can potentially diminish its effective orifice. Our TEE examinations, however, did show low prosthetic valve gradients. The thickness of the metal ring itself is less than 1 $\mathrm{mm}$, and the thickness of the textile cloth can be further diminished. The prosthetic valves that were used were not specifically manufactured for the rings; it can be foreseen that in the final product the ring and valve will be

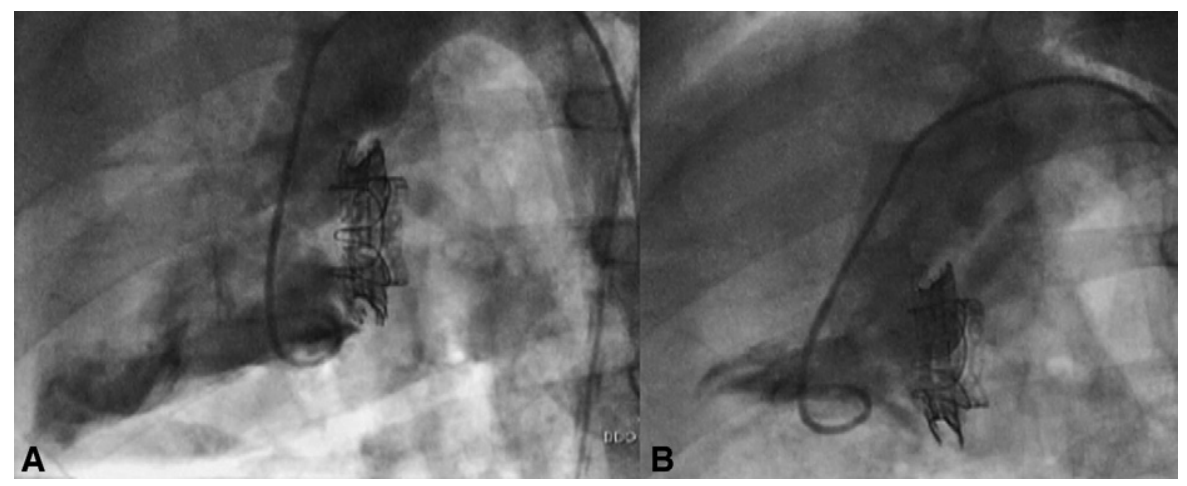

FIGURE 3. Device in mitral position at diastole (A) and systole (B) on left ventricular angiogram. 


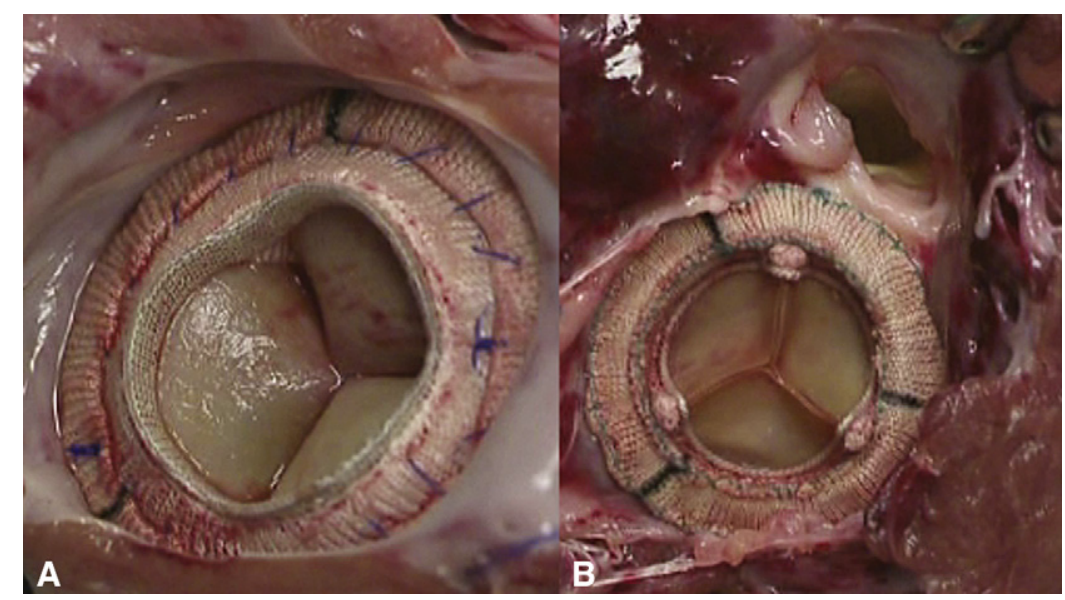

FIGURE 4. Device in mitral position from left atrial side (A) and left ventricular side (B) at autopsy.

manufactured as one unit, which can be further optimized, also in respect to the distance that the valve prosthesis is positioned inward the left atrium, and to the shape and thickness of the valve struts. Placement of the valve prosthesis on top (supra-annular) of the attachment ring means that it is positioned higher inside the left atrium, resulting in a lower risk for LVOT obstruction, as can sometimes be observed with biological prostheses that are hand sewn. ${ }^{10}$ One can also argue that use of a single annular suture means that the method is in fact not fully sutureless. However, such suture is not connected directly to the device. Also, Magovern and associates ${ }^{11,12}$ used such a single annular suture in many of the clinical cases of aortic or mitral valve replacement with the Magovern sutureless prosthetic valve. It is not clear yet whether such suture will be necessary in the human situation, where the mitral valve annulus will be more pathologically degenerated and rigid by calcifications, than in the young and healthy pigs we used in these acute experiments. It is also conceivable to stiffen and/or narrow the natural valve annulus with other sutureless methods in the future.

The current still experimental mitral valved stents ${ }^{4-6}$ have more difficulty with attaching themselves solidly to the mitral valve annulus and still have a relatively higher risk for paravalvular leakage. The difference between a valved stent and our Nitinol ring is that the valved stent loses its radial expansion force as soon as the valve prosthesis inside the stent has fully expanded, whereas the force between the flanges of our Nitinol ring will remain active, independent of the grade of expansion of the valve prosthesis. Another disadvantage of valved stents is that because they need their radial expansion to attach, in the often dilated mitral valve annulus, very large stent diameters with large valve diameters will be required. Expansion of such dilated annulus by a valved stent may compress the LVOT and distort the aortic valve ostium, or the lower side of the stent may cause LVOT obstruction. All these theoretical disadvantages may be prevented by using Nitinol rings, which have a fixed diameter, have a relatively short height, and use axial compression. It is foreseeable in the future that our devices can be crimped similar to valved stents, but that they will attach themselves with a stronger adhesive force than that of (current) valved stents. We are currently developing an applicator that can hold and constrain the device mechanically, preventing early expansion of the lower

TABLE 2. Outcome

\begin{tabular}{lcccc}
\hline Case no. & Weaned from ECC & MR LV angio/TEE & LVOTO LV angio/TEE & Device at autopsy \\
\hline $1^{*}$ & Yes & $2+$ & 0 & Tilted \\
2 & No & 0 & 0 & Good position, no leakage \\
$3^{*}$ & Yes & 0 & 0 & Dislocated \\
4 & Yes & 0 & 0 & Good position, no leakage \\
5 & Yes & 0 & 0 & Good position, no leakage \\
6 & Yes & - & - & Good position, no leakage \\
7 & Yes & 0 & 0 & Good position, no leakage \\
$8 \dagger$ & Yes & $4+$ & 0 & Dislocated \\
9 & Yes & 0 & 0 & Good position, no leakage \\
\hline
\end{tabular}

ECC, Extracorporeal circulation; $M R$, mitral regurgitation; TEE, transesophageal echocardiography; $L V$, left ventricle; $L V O T O$, left ventricular outflow tract obstruction. *Operated on fibrillating heart. † $\nmid$ Large atrial septal defect; device malpositioned. 
flange when in contact with warm blood, and allowing its use in a fibrillating or beating heart too. Further long-term animal testing will be necessary to prove the feasibility of this method to replace the mitral valve suturelessly.

Some parts of the prototypes were provided by Edwards Lifesciences, Ltd (Irvine, Calif), Endosmart GmbH (Stutensee, Germany), Jotec GmbH (Hechingen, Germany), and St Jude Medical, Inc, St Paul, Minn. The videos were produced by Mediaventures, Merelbeke, Belgium.

Besides the authors, the following persons contributed to the Acute Sutureless Mitral Valve Project (in alphabetical order): Yaroslava Dmitrieva, MD (cardiologist, Herzzentrum Leipzig, Germany), Grit Gerullis (veterinarian assistant, Herzzentrum Leipzig, Germany), Yme Groeneveld (EPS, The Hague, Netherlands), Olliver Gross (perfusionist, Herzzentrum Leipzig, Germany), Jochen Hahn, MD (surgical assistant, Herzzentrum Leipzig, Germany), Pierre Janssen (Instrumentation Department Catharina Hospital, Netherlands), Willem-Jan Janssen (Edwards Lifesciences, Netherlands), Astrid Jorna (St Jude, Netherlands), Henk van Kemenade (Van Kemenade Slaughterhouse, Netherlands), Sara Klein (veterinarian assistant, Herzzentrum Leipzig, Germany), Marjan van Marle (traffic, Netherlands), Hardy Mueller (Jotec GmbH, Germany), Thomas Mueller (perfusionist Herzzentrum Leipzig, Germany), Steven Noulet (Mediaventures, Belgium), Mike Roelofs (Audiovisual Department, Catharina Hospital, Netherlands), Carl Swindle (Edwards Lifesciences, Irvine, Calif), Guy van Dael (Audiovisual Department Catharina Hospital, Netherlands), Wim Van Renterghem (Mediaventures, Belgium), and Veid Zebralla MD (surgical assistant Herzzentrum Leipzig, Germany).

\section{References}

1. Andersen HR, Knudsen LL, Hasenkam JM. Transluminal implantation of artificial heart valves. Description of a new expandable aortic valve and initial results with implantation by catheter technique in closed chest pigs. Eur Heart J. 1992;13:704-8.

2. Cribier A, Eltchaninoff H, Bash A, Borenstein N, Tron C, Bauer F, et al. Percutaneous transcatheter implantation of an aortic valve prosthesis for calcified aortic stenosis. Circulation. 2002;106:3006-8.

3. Hanzel GS, O'Neill WW. Complications of percutaneous aortic valve replacement: experience with the Cribier-Edwards percutaneous heart valve. EuroInterv Suppl. 2006;1:A3-8.

4. Ma L, Tozzi P, Huber C, Taub S, Gerelle G, von Segesser LK. Double-crowned valve stents for off-pump mitral valve replacement. Eur J Cardiothorac Surg. 2005;28:194-9.

5. Lutter G, Quaden R, Osaki S, Hu J, Renner J, Edwards NM, et al. Off-pump transapical mitral valve replacement. Eur J Cardiothorac Surg. 2009;36:124-8.

6. Lozonschi L, Quaden R, Edwards NM, Cremer J, Lutter G. Transapical mitral valved stent implantation. Ann Thorac Surg. 2008;86:745-8.

7. Berreklouw E, Vogel B, Fischer H, Weinberg F, van Rijk-Zwikker GL, Van Nooten GJ, et al. Fast sutureless implantation of mechanical aortic valve prostheses using Nitinol attachment rings: feasibility in acute pig experiments. J Thorac Cardiovasc Surg. 2007;134:1508-12.

8. Berreklouw E, Koene B, De Somer F, Bouchez S, Chiers K, Taeymans Y, et al. Sutureless replacement of aortic valves with St Jude Medical mechanical valve prostheses and Nitinol attachment rings: feasibility in long-term (90-day) pig experiments. J Thorac Cardiovasc Surg. 2010 Aug 19. In press. [Epub ahead of print].

9. Sellers RD, Levy MJ, Amplatz K, Lillehei CW. Left retrograde cardioangiography in acquired cardiac disease; technic, indications and interpretations in 700 cases. Am J Cardiol. 1964;14:437-47.

10. Bortolotti U, Milano A, Tursi V, Minarini M, Thiene G, Mazzucco A. Fatal obstruction of the left ventricular outflow tract caused by low-profile bioprosthes in the mitral valve position. Chest. 1993;103:1288-9.

11. Magovern GJ, Kent EM, Cromie HW, Cushing WB, Scott S. Sutureless aortic and mitral prosthetic valves. Clinical results and operative technique on sixty patients. J Thorac Cardiovasc Surg. 1964;48:346-61.

12. Magovern GJ, Liebler GA, Park SB, Burkholder JA, Sakert T, Simpson KA Twenty-five-year review of the Magovern Cromie sutureless aortic valve. Ann Thorac Surg. 1989;48:S33-4. 


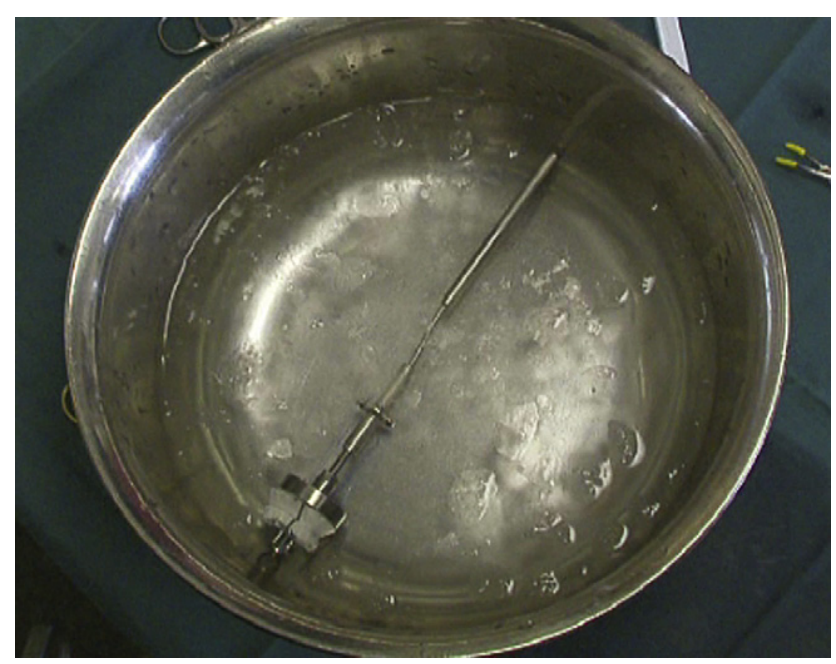

FIGURE E1. Device loaded and stretched on applicator in ice water.

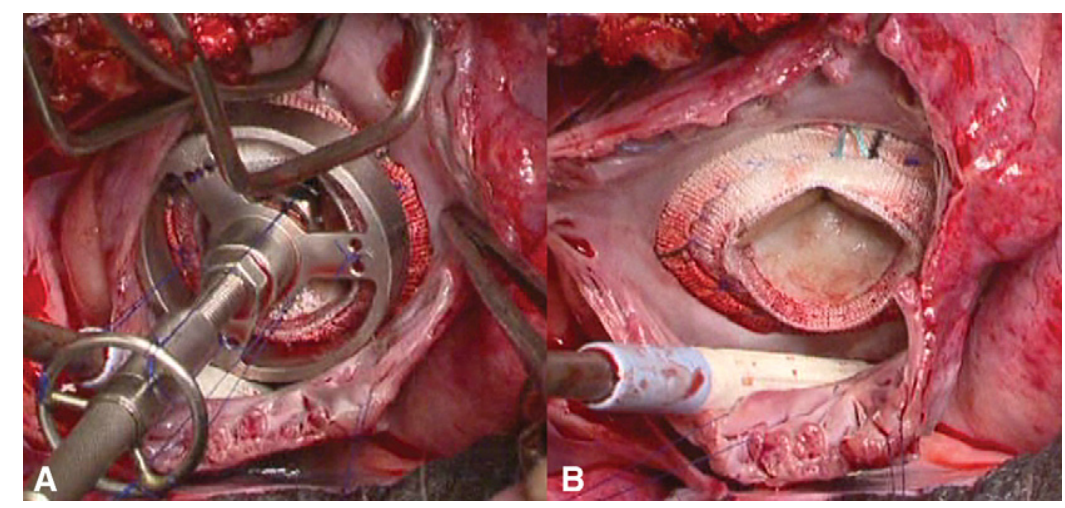

FIGURE E2. A, Device and applicator positioned in mitral valve annulus before activation. B, Device positioned in mitral valve annulus after activation. 